\title{
Thermocapillary Convective Flows Generated by Laser Points or Patterns: Comparison for the Non-Contact Micromanipulation of Particles at the Interface
}

\author{
Ronald Terrazas Mallea ${ }^{1,2}$, Dimitri Piron ${ }^{1}$, Aude Bolopion ${ }^{2}$, Pierre Lambert ${ }^{1}$, Michael Gauthier ${ }^{2}$
}

\begin{abstract}
This work compares the controlled manipulation of micrometer size particles using thermocapillary convective flows generated by laser points or patterns. Laser-induced thermocapillary convective flows are a promising solution to manipulate particles at the interface. These flows are generated when a surface tension stress is generated at the fluid/gas interface due to a thermal gradient. Laser heating allows to generate fast, localized flows that are used to displace a particle towards a desired position. However, these flows are repulsive and so the system is unstable. Although using the simple laser spot with a closedloop control enables to control the particle displacement, the control of the particle movement direction is somehow difficult and the particle position stabilization remains challenging. In this paper, it is proposed to use laser patterns in order to overcome these limitations. Experimental tests are performed using a 500$\mu \mathrm{m}$-diameter steel spherical particle that is displaced towards a target position. The preliminary experimental results show that it is possible to overcome the above mentioned limitations while still obtaining maximal particle velocities ranging from 4 to 9 $\mathrm{mm} / \mathrm{s}$ during the displacement phase.
\end{abstract}

Index Terms-Automation at Micro-Nano Scales, Motion Control, Visual Servoing.

\section{INTRODUCTION}

$\mathbf{T}$ HE last few years have seen an increasingly growing interest for non-contact actuation of micrometer size objects. In vivo medical procedures involving magnetically actuated microrobots [1], in vitro cell sorting performed by controlled dielectrophoresis [2] or even microassembly of artificial objects are potential application fields [3].

Lasers are also widely used. Optical tweezers use the radiation pressure of the light to move objects [4]. The particularity of this actuation principle is that the force applied by the

Manuscript received: February, 23, 2018; Revised April, 23, 2018; Accepted June, 18, 2018.

This paper was recommended for publication by Editor Yu Sun upon evaluation of the Associate Editor and Reviewers' comments. This work was supported by Interuniversity Attraction Poles Programme (IAP 7/38 MicroMAST) initiated by the Belgian Science Policy Office, by the Labex ACTION project (contract "ANR-11-LABX-01-01") and by the Région Franche-Comté.

${ }^{1}$ Ronald Terrazas Mallea, Dimitri Piron and Pierre Lambert are with the TIPs department CP 165/67, Ecole Polytechnique de Bruxelles, Universite Libre de Bruxelles, 1050 Bruxelles, Belgium. rterraza@ulb.ac.be, pierre. lambert@ulb.ac.be

2 Ronald Terrazas Mallea, Aude Bolopion and Michael Gauthier are with the FEMTO-ST Institute, AS2M department, Univ. Bourgogne FrancheComté, Univ. de Franche-Comté/CNRS/ENSMM, 24 rue Savary, F-25000 Besançon, France aude.bolopion@femto-st.fr

Digital Object Identifier (DOI): see top of this page. laser is short range. A lot of research has been done for this type of manipulation. To mention some results, in [5] a 3D force sensing method has been developed and used in an haptic interface to manipulate particles in 3D. In [6], a path planning method is proposed that can achieve scalable and collision-free manipulation of multiple particles in parallel. In [7], cells are manipulated indirectly using silica beads arranged in different formations. Several objects can be controlled by switching the laser from one object to another at high speed [8], or by using holographic patterns of light to produce several independent traps [9]-[12]. Moreover, laser patterns can be used to provide additional functionalities. For example, the particles can also be rotated using special pattern shapes. This has been achieved using a spiral interference pattern [13], using multiple beams on the same particle [14] or using a 5th order Bessel shaped beam [15]. The beam intensity distribution can also be modified as in [16], where a crescent-shaped beam is used to trap the particle with reduced intensity at the particle center to avoid damaging the particle nucleus.

Alternatively, heat produced by the laser can be exploited to onset thermal-driven convective flows on a container filled with liquid to transport objects. These convective flows can be sorted into two categories: natural and Marangoni convection if the liquid is heated from below [17], [18], or thermocapillary convection if heated from atop. These two types of convection for particle manipulation were compared previously [19].

Thermocapillary convection has already been used in different manners. In [20], [21] it is used to displace liquid droplets. In [22], [23], the thermocapillary convective flow is generated by heating the bottom of a bubble interface using a light pattern inside a closed chamber filled with a fluid. In [24] and [25], the thermocapillary convective flow is generated around bubble microrobots which are created by heating a laser-light absorbent substrate. However these bubbles were small and dissipated when the laser was turned off. A different approach is used in [26]-[28], where the bubble microrobots are created by increasing the laser power temporarily and reduced to operating level as soon as a large enough bubble is created which remains in the workspace permanently. However in these last works, the flow around the bubble is not used to displace the particle but the bubble itself. So the bubble is used to push the particles towards the target locations.

Laser patterns have also been applied to systems using the thermocapillary flows to manipulate particles. In [22], 
[23], [25]-[28], laser patterns are used to generate patterns of multiple points which generate/actuate multiple bubble microrobots which are then used to manipulate the particles. As the particles are pushed or attracted by the bubble, the control of these systems is quite simple. However, the manipulation velocities are restricted to few hundreds of $\mu \mathrm{m} / \mathrm{s}$.

Different from the cited works, the system used in this paper generates the thermocapillary convective flow by directly heating the water/air interface using a laser. The physical principle was presented in details in [19] where initial experiments in open-loop were performed. The results showed that $500 \mu \mathrm{m}$ particles could be displaced at velocities up to $5 \mathrm{~mm} / \mathrm{s}$ which is around 10 times faster than the fastest velocity reported using thermocapillary flows [22]. Such velocities are possible because, according to the Stokes's law, the particle should move as fast as the fluid velocity in steady-state.

Using the information about the flow characteristics, a model for the system was proposed and used to design a closed-loop controller [29]. Experiments were performed with a $500 \mu \mathrm{m}$ diameter AISI 304 steel particle displaced towards a target location with velocities between 4-9 mm/s. However, stabilizing the particle position at the target location was a problem due to the repulsive nature of the flow which makes the system intrinsically unstable. In addition, point based control requires two separated controllers to control respectively the magnitude of the particle velocity and the particle velocity direction. To solve these problems, two laser patterns are proposed instead. An arc laser pattern is used during the displacement phase to facilitate the control of the particle velocity direction allowing to control it using only an open-loop controller. Then a circular pattern is used to trap the particle inside the circle, stabilizing the particle position inside the tolerance region.

This paper is organized as follows: in Section II, the actuation principle is presented together with the experimental setup. In Section III, the control strategy using laser points is described and its limitations are highlighted. In Section IV, the system is characterized using laser patterns which shows the advantages of using them. Section $\mathrm{V}$ presents the light pattern based control strategy, the preliminary results and discusses the proposed approach. Section VI concludes the paper.

\section{ACTUATION PRINCIPLE AND EXPERIMENTAL SETUP}

\section{A. Actuation principle}

Details about the physics governing the actuation based on thermocapillary convective flows can be found in [19], [30]. A container filled with water is initially at equilibrium (the fluid velocity is null) and at ambient temperature. Then, a collimated laser beam locally heats the water. The temperature gradient at the interface generates a surface tension stress profile that is compensated by a viscous stress. This onsets a fluid motion, named thermocapillary convective flow, across the entire fluid according to the Navier-Stokes' equation [19] which goes outwards the laser spot and is significant only close to it. A particle lying at the air/liquid interface is affected by the thermocapillary convective flow and it moves along the flow direction.

\section{B. Experimental Setup}

The experimental setup used to generate and control these thermocapillary convective flows is depicted in Figure 1. The laser system is composed of a continuous-wave $1455 \mathrm{~nm}$ infrared laser (Keopsys Fiber Raman Laser). The power of this laser can be adjusted up to $1 \mathrm{~W}$. It is used to heat the air/liquid interface. As it is not visible, it is coupled with a continuous-wave $655 \mathrm{~nm}$ red laser using a laser coupler to detect its position in the workspace. The coupled beam is collimated using a lens with focal length of $6.24 \mathrm{~mm}$ (Thorlabs lens A110TM). The collimated laser beam is directed towards a plastic container filled with distilled water, using a 2DOF piezo-actuated tip/tilt mirror (Mirrorcle Technologies A7M20.1) which has a mechanical tip/tilt range of $\pm 87 \mathrm{mrad}$ on each axis (equivalent to $\pm 174 \mathrm{mrad}$ optical beam deflection). The mirror is connected to the controller Mirrorcle Technologies USB-SL-M4-B160, which allows to linearly control the mirror deviation using programmed routines. The mirror has a response time of $1 \mathrm{~ms}$ when operating in pointto-point mode or a scanning frequency of 50000 instructions per second when operating in the data stream mode. This mode is used to scan the arc or the circular pattern 100 times per second. This means that a pattern is scanned in $10 \mathrm{~ms}$ which is 80 times faster than the $0.8 \mathrm{~s}$ taken by the particle to reach steady-state velocity [29]. Thus it is considered that the patterns are scanned fast enough to be considered continuous w.r.t. the thermocapillary system.

The experiments are performed using stainless steel AISI 304 spherical particles with a diameter of $500 \mu \mathrm{m}$ (Redhill Precision), which are deposited at the water surface. According to the wetting conditions, the particle can float and reach a certain equilibrium position (a detailed analysis is performed in [31]). The water layer thickness is around $7.5 \mathrm{~mm}$ contained on a square plastic container with dimensions of $115 \times 115$ $\mathrm{mm}$. The container is covered using a plastic lid in order to reduce the surface contamination through time. The container is on top of a PHLOX white led back light LEDW-BL100x100-LLUB-Q-1R-24V, which provides a uniform background illumination that facilitates the image recognition. The particle position is tracked using with an IDS camera (UI$3370 \mathrm{CP}$ ) with a resolution of $1024 \times 1024$ pixels at a frame rate of 10 FPS. The camera is tilted $1.8^{\circ}$ in order to have vision of the whole working space. In order to reduce the infrared light reflection coming to the camera sensor, an absorptive neutral density filter (Thorlabs NENIR40BC) is placed on top of it. For the camera optics, a $50 \mathrm{~mm}$ focal length lens is used together with a $10 \mathrm{~mm}$ extensor ring. The field of view is adjusted to be $65 \times 65 \mathrm{~mm}$ (one pixel represents $63.5 \mu \mathrm{m}$ ).

\section{PARTiCle MOTION CONTROL USING LASER POINTS}

\section{A. Control strategy using laser points}

Actuation using thermocapillary convective flows generated by laser points has been reported in [19], [29] and closed-loop control has been implemented in [29], which is summarized here below.

As shown in Fig. 2, the physical system input is the desired laser position $\mathbf{X}_{\text {lasD }}$ and its output is the particle velocity $\dot{\mathbf{X}}_{\text {part }}$ 


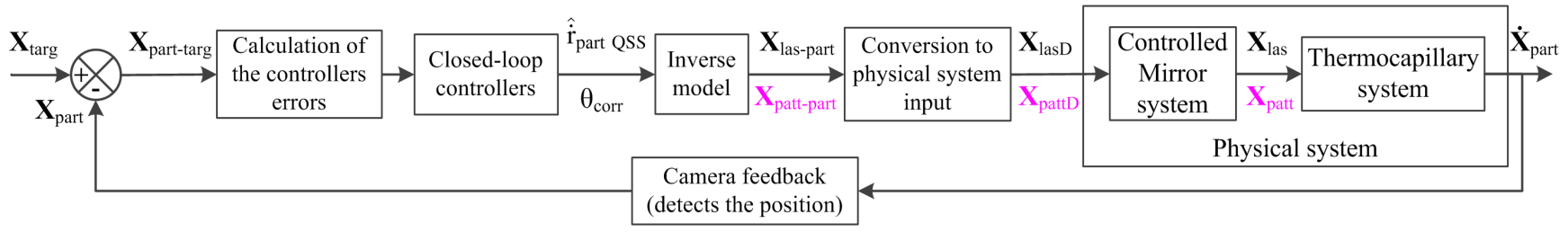

Fig. 2: Simplified block diagram representation of the entire closed-loop system. The physical system to control is composed by the Controlled Mirror system and the Thermocapillary system. Defined as such, the physical system has as input a desired laser position $\mathbf{X}_{\text {lasD }}$ and as output the particle velocity $\dot{\mathbf{X}}_{\text {part }}$. The required value of $\mathbf{X}_{\text {lasD }}$ to displace the particle position $\mathbf{X}_{\text {part }}$ towards a target position $\mathbf{X}_{\text {targ }}$ is defined by two closed-loop controllers. Based on the current error, these controllers compute two outputs: an estimated particle velocity at quasi steady-state $\hat{\dot{r}}_{\text {part }}$ QSS and a correction angle $\theta_{\text {corr }}$ which are then transformed into the actual input of the physical system $\mathbf{X}_{\text {lasD }}$. This controller structure is the same when using the arc pattern. It is just necessary to replace the laser point related variables by the pattern variables which are: the pattern position $\mathbf{X}_{\text {patt }}$, the desired pattern position $\mathbf{X}_{\text {patt }}$ and the pattern-particle position $\mathbf{X}_{\text {patt-part }}$. These variables are written in magenta.

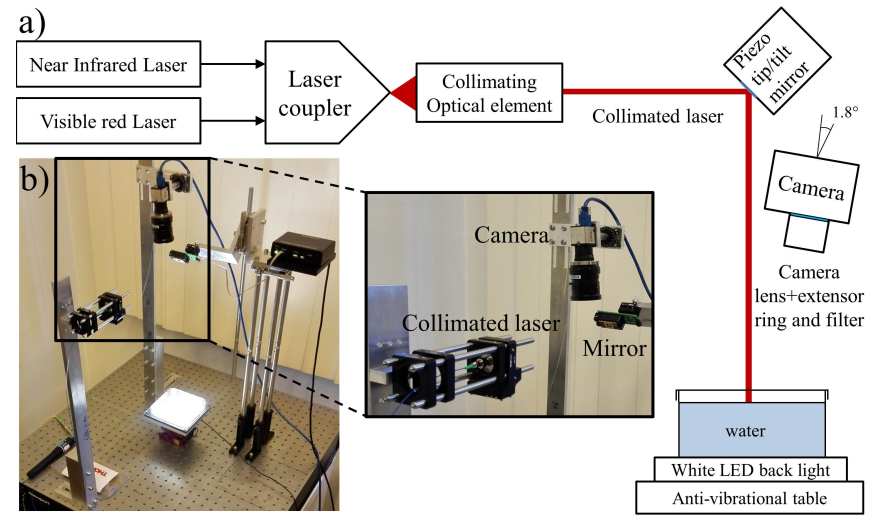

Fig. 1: Experimental setup for non-contact actuation using thermocapillary convective flows.

with magnitude $\dot{r}_{\text {part }}$ and direction $\theta_{\text {part }}$. As this system system is non-linear and coupled, inversion based control is performed so that the system output can be decoupled and linearized with respect to the inputs. As a result, two single input single output (SISO) systems are defined.

The first SISO system defines the particle velocity magnitude $\dot{r}_{\text {part }}$ and has as input the estimated particle velocity at quasi steady-state $\hat{\dot{r}}_{\text {part }}$ Qss. This is done based on a experimentally identified relationship. The second SISO system defines the particle velocity direction $\theta_{\text {part }}$ and has as input a correction angle $\theta_{\text {corr }}$.

Two controllers are therefore defined. The particle velocity magnitude controller controls the first SISO system. This controller computes an estimated particle velocity at quasi steady-state $\hat{\dot{r}}_{\text {part }}$ QSS in function of an input which is the particle-target distance $r_{\text {part-targ }}$. A PD (proportional derivative) controller is implemented in this case.

The particle velocity direction controller controls the second SISO system. This controller computes a correction angle $\theta_{\text {corr }}$ in function of an input which is the difference between the particle-target orientation $\theta_{\text {part-targ }}$ and the current particle velocity direction $\theta_{\text {part }}$. A proportional controller is implemented in this case.

\section{B. Previous results and manipulation limitations}

In [29], a $500 \mu \mathrm{m}$ diameter steel spherical particle is displaced towards the vicinity of a target position. When the position enters a tolerance region defined around the target position, actuation is stopped (the laser is placed far enough from the particle so that the flow does not affect the particle). Maximal velocities up to $4-9 \mathrm{~mm} / \mathrm{s}$ are attained during the control phase, which can be compared with the fastest manipulation techniques that use Marangoni effect.

Despite these interesting results two limitations arise. The first major issue is the complexity of the control. Two independent controllers are needed, to control respectively the particle velocity magnitude and its direction. In addition, limited performances are obtained using the particle velocity direction controller, since the error in direction reaches about $20^{\circ}$ when operated at full speed (Fig. 3a). As the velocity decreases, the control of the particle becomes more difficult since the trajectory of the particle is perturbed by undesired fluid flows. This means that the noise causes the particle moving randomly (errors up to $\pm 90^{\circ}$ can be measured)

The second major issue is the stabilization of the particle inside the tolerance region (see Fig. $3 b$ and 4 ). When the particle enters the tolerance region actuation is stopped. However, the particle bounces back, exiting the tolerance region. The controllers are turned on again, but as soon as they are stopped the particle exists the tolerance region again. This "bouncing back effect" seems to occur due to a counter flow that is generated when the laser stops heating the region close to the particle. When this happens, some cold flow is still dragged to the surface due to fluid inertia. However as the laser is not heating anymore, this fluid is colder than the fluid surrounding it. This generates a thermocapillary convective flow going inwards the position where the laser was which pulls the particle backwards. This phenomenon makes very difficult to stabilize the particle position using this control strategy as the particle would be constantly going in and out the tolerance region.

This paper solves the two mentioned issues using laser patterns. An arc pattern is proposed to ensure a better control over the particle velocity direction making the overall control easier. A circular pattern is envisioned to ensure the particle 


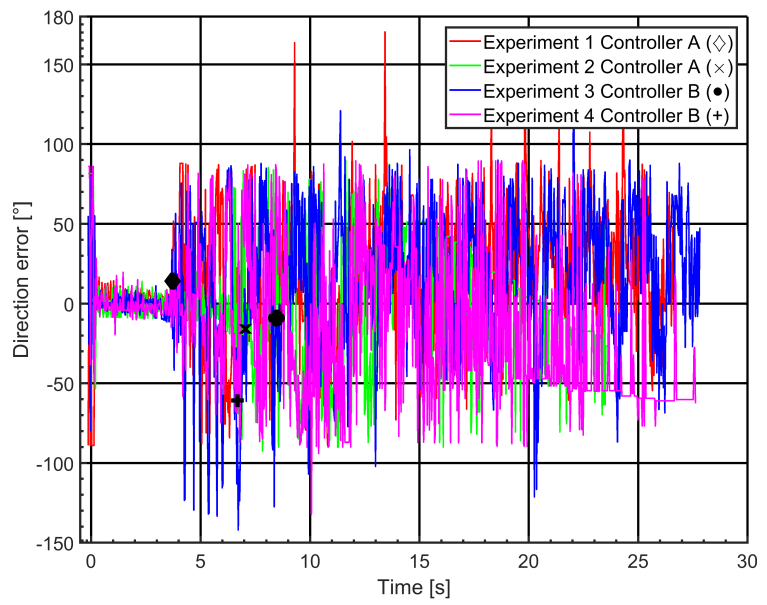

(a) Direction of the particle displacement.

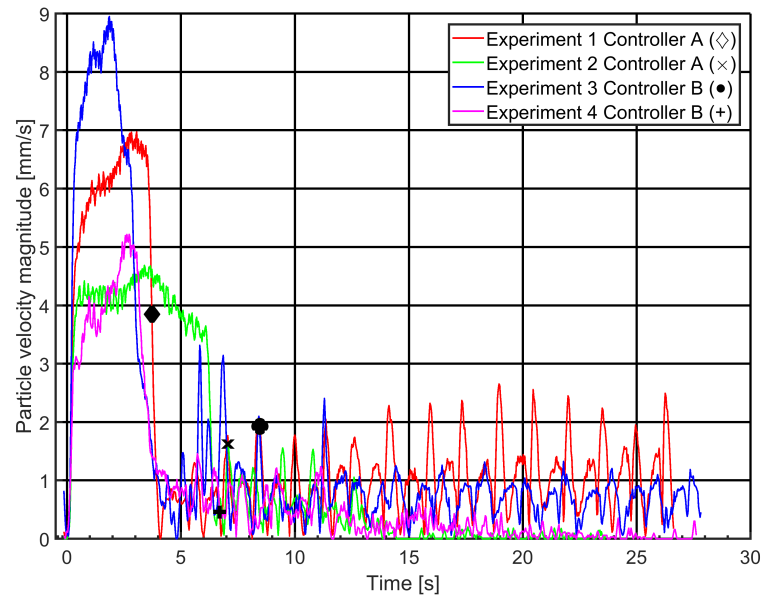

(b) Particle velocity magnitude in function of time.

Fig. 3: Two particle velocity magnitude controllers are defined, as detailed in [29]: the first one (Controller A) is set in order to get a settling time of $2 \mathrm{~s}$ and an overshoot of $10 \%$. The second one (Controller B) is set to have a settling time of $2 \mathrm{~s}$ and an overshoot of $1 \%$. The markers represent the time instant when the particle first enters the tolerance region.

position stabilization. Details are given in in the following sections.

\section{THERMOCAPILLARY SYSTEM CHARACTERIZATION USING LASER PATTERNS}

\section{A. Characterization of the arc pattern parameters}

1) Definition of the arc pattern: To control the particle velocity direction during its displacement, an arc pattern is proposed, defined by the parameters shown in Figure 5. We characterized the new actuation principle by studying the influence of the pattern parameters on the velocity and on the particle movement deviation. The objective is twofold:

- maximise the velocity to reduce manipulation time,

- decrease the particle deviation due to instabilities.

As shown in Figure 5, the arc is defined by three parameters: the pattern radius $R_{\text {patt }}[\mathrm{mm}]$, the pattern aperture angle $\theta_{\text {patt }}$ $\left[{ }^{\circ}\right]$ and the laser power $P_{\text {las }}[\mathrm{mW}]$.

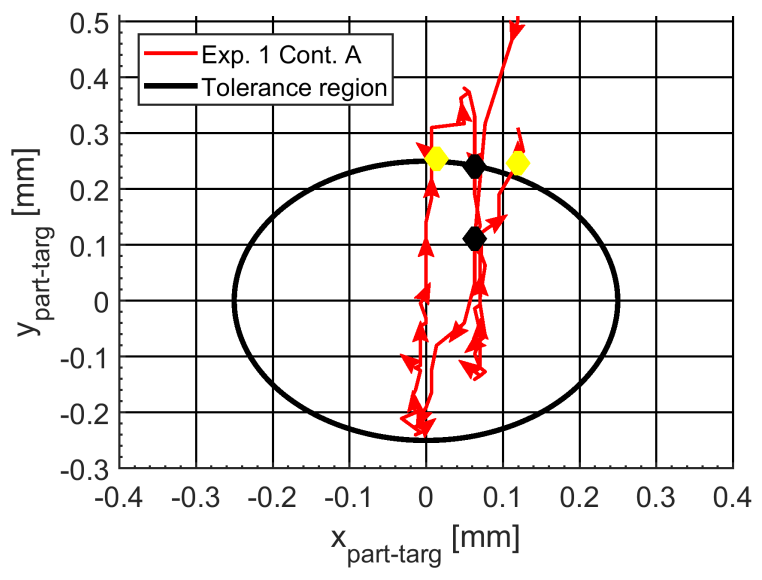

Fig. 4: Trajectory of the particle in the vicinity of the tolerance region. The yellow and black markers indicate the positions at which the controllers were turned on and off respectively.

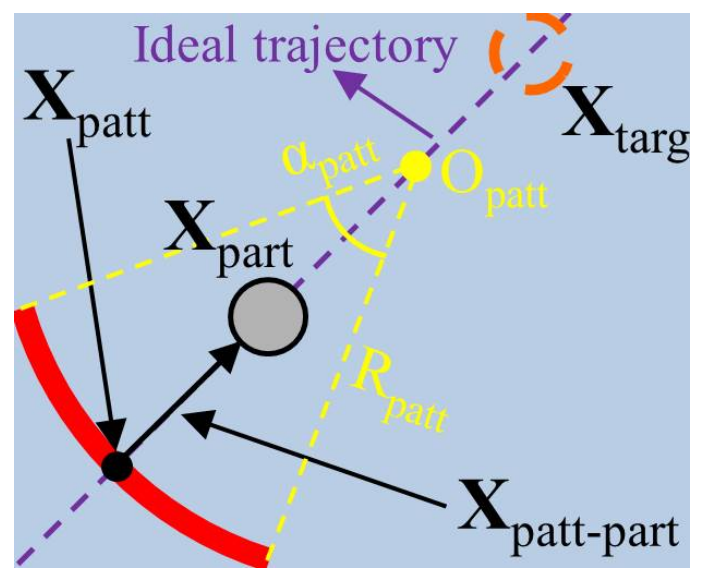

Fig. 5: Representation of the geometrical parameters of the arc laser pattern. The purple line represents the ideal trajectory the particle should follow without the presence of perturbations.

To ensure that a symmetric flow acts on the particle, the arc pattern axis of symmetry must always contain the particle. To guarantee this, the particle position $\mathbf{X}_{\text {part }}$ and the pattern center position $\mathbf{X}_{\text {patt }}$ must be collinear (Fig. 5). In this way, the pattern-particle distance $r_{\text {patt-part }}$ can be tunned by translating the pattern along the axis of symmetry. The pattern-particle orientation $\theta_{\text {patt-part }}$ can be tunned by rotating the pattern around the particle. This allows to use $\mathbf{X}_{\text {patt-part }}$ as the actuation variable for the control strategy in the same form as in the previous case with the laser points [29].

2) Analysis of the parameters of the arc pattern: For the characterization of the arc pattern parameters, the patternparticle distance $r_{\text {patt-part }}$ is maintained constant during all the experiments. Furthermore, in order to measure the movement deviation, the orientation of the arc pattern will remain constant during all experiments. A target location $\mathbf{X}_{\text {targ }}$ is set and the laser pattern position $\mathbf{X}_{\text {patt }}$ will move along a straight line towards the target. Indeed, in the ideal case the particle should move in the direction of the pattern-particle vector $\mathbf{X}_{\text {patt-part }}$. In this case, this means the particle should also move in the straight line connecting the laser position with 
the target position. Consequently, the deviation is defined as the displacement in the perpendicular direction to that ideal trajectory.

Each experiment associated to a given set of fixed parameters is performed four times. Results of the four experiments are averaged to expose the results trend and cancel out noise effects. The obtained results are shown in table I.

TABLE I: Particle velocity and deviation in function of the pattern radius $R_{\text {patt }}$, the aperture angle $\alpha_{\text {patt }}$ and the laser power $P_{\text {las }}$.

\begin{tabular}{|c|c|c|c|c|c|}
\hline & $\begin{array}{l}\text { Radius } \\
{[\mathrm{mm}]}\end{array}$ & $\begin{array}{c}\text { Aperture } \\
{\left[{ }^{\circ}\right]}\end{array}$ & $\begin{array}{l}\text { Power } \\
{[\mathrm{mW}]}\end{array}$ & $\begin{array}{l}\text { Velocity } \\
{[\mathrm{mm} / \mathrm{s}]}\end{array}$ & $\begin{array}{l}\text { Deviation } \\
{[\mathrm{mm}]}\end{array}$ \\
\hline \multirow{3}{*}{$\begin{array}{l}\text { Radius } \\
\text { variation }\end{array}$} & 3.22 & \multirow{3}{*}{180} & \multirow{3}{*}{120} & 8 & 4.6 \\
\hline & 4.83 & & & 4 & 5.7 \\
\hline & 6.44 & & & 3 & 2.5 \\
\hline \multirow{3}{*}{$\begin{array}{l}\text { Aperture } \\
\text { variation }\end{array}$} & \multirow{3}{*}{3.22} & 180 & \multirow{3}{*}{120} & 8 & 4.6 \\
\hline & & 122.4 & & 8.7 & 9.5 \\
\hline & & 57.6 & & 7.6 & 7.9 \\
\hline \multirow{3}{*}{$\begin{array}{l}\text { Power } \\
\text { variation }\end{array}$} & \multirow{3}{*}{3.22} & \multirow{3}{*}{180} & 120 & 8 & 4.6 \\
\hline & & & 100 & 7 & 3.3 \\
\hline & & & 80 & 3.5 & 2.2 \\
\hline
\end{tabular}

The first parameter being fixed is the aperture angle $\alpha_{\text {patt }}$, to $180^{\circ}$. Indeed, it allows to operate at high velocities while reducing the deviation which is especially advantageous and is discussed in more details in the next subsection. For $R_{\text {patt }}$ and $P_{\text {las }}$, two possibilities are available: set $R_{\text {patt }}$ and $P_{\text {las }}$ to $6.44 \mathrm{~mm}$ and $120 \mathrm{~mW}$ respectively or to $3.22 \mathrm{~mm}$ and 120 $\mathrm{mW}$. The first possibility offers the least particle deviation $(2.5$ $\mathrm{mm})$ but also reduces the attained velocity. The second choice offers the maximum velocity $(8 \mathrm{~mm} / \mathrm{s})$ at the cost of having a larger deviation. Since the reduction of the manipulation time is a critical asset for the non-contact micromanipulation using thermocapillary convection, the second set of parameters is selected. This means the arc parameters to use are: $R_{\text {patt }}=$ $3.22 \mathrm{~mm}, \theta_{\text {patt }}=180^{\circ}$ and $P_{\text {las }}=120 \mathrm{~mW}$.

\section{B. Particle velocity direction analysis}

One of the main limitations of the actuation using laser points was that the particle velocity direction $\theta_{\text {part }}$ had to be controlled in closed-loop. Ideally, the particle should move in the direction of the laser-particle vector $\mathbf{X}_{\text {las-part }}$ or for the patterns, in the direction of the pattern-particle vector $\mathbf{X}_{\text {patt-part }}$. So if the laser spot or pattern is placed at the bottom of the particle, the particle should move upwards in a straight line.

Having this in mind, let us compare the results obtained using different arc pattern apertures and also the laser spot in open-loop. Fig. 6 shows the trajectories followed by the particle when the laser point/pattern was placed at the bottom of the particle and then moved upwards. Using the arc pattern, the particle velocity direction $\theta_{\text {part }}$ tends to the pattern-particle direction $\theta_{\text {patt-part }}$ faster and with more accuracy than using the laser spot alone. The best results is obtained using the arc pattern aperture of $180^{\circ}$ for which the standard deviation of the difference between these two values was below $1^{\circ}$. With such small variation, an open-loop controller is enough to control the particle velocity direction using the arc pattern.

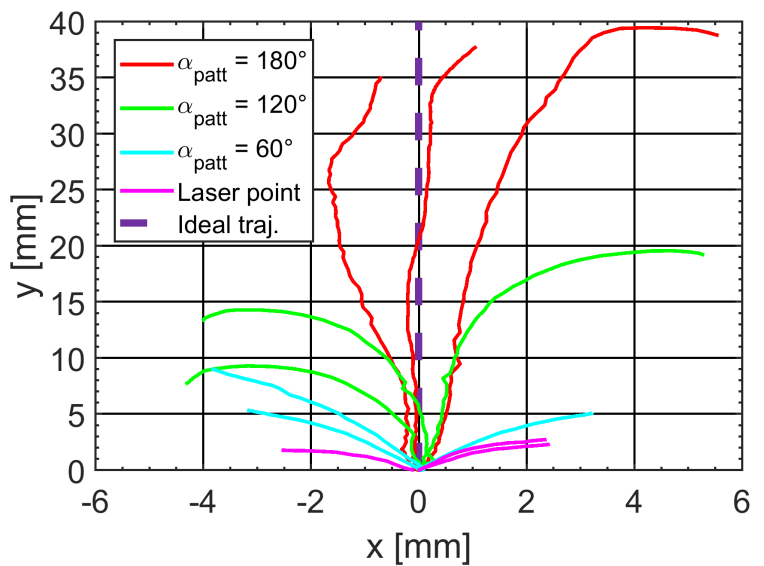

Fig. 6: Particle deviation when using the arc pattern and the simple laser spot for different aperture angles $\alpha_{\text {patt }}$. The arc pattern radius $R_{\text {patt }}$ had a value of $3.22 \mathrm{~mm}$ for all the experiments.

\section{Particle velocity magnitude identification}

A similar control strategy as the one used for the laser points is used for the laser patterns. This requires identifying an inverse model relating an estimated particle velocity at quasi steady-state $\hat{\dot{r}}_{\text {part }}$ QSS with the pattern-particle distance $r_{\text {patt-part }}$. For this purpose, the same identification method as the one presented in [29] is used.

Five pattern-particle distances are tested: $r_{\text {patt-part }}=1.0,1.25$, $1.5,1.75,2.0 \mathrm{~mm}$. For each distance, the pattern is placed at the top, right, bottom, and left of the particle $\left(\theta_{\text {patt-part }}=0^{\circ}, 90^{\circ}\right.$, $180^{\circ}, 270^{\circ}$ ). Each $r_{\text {patt-part }}$ value is tested in four different water samples, 8 tested eight times in total using each orientation two times. This means that each $r_{\text {patt-part }}$ value is tested 32 times in total.

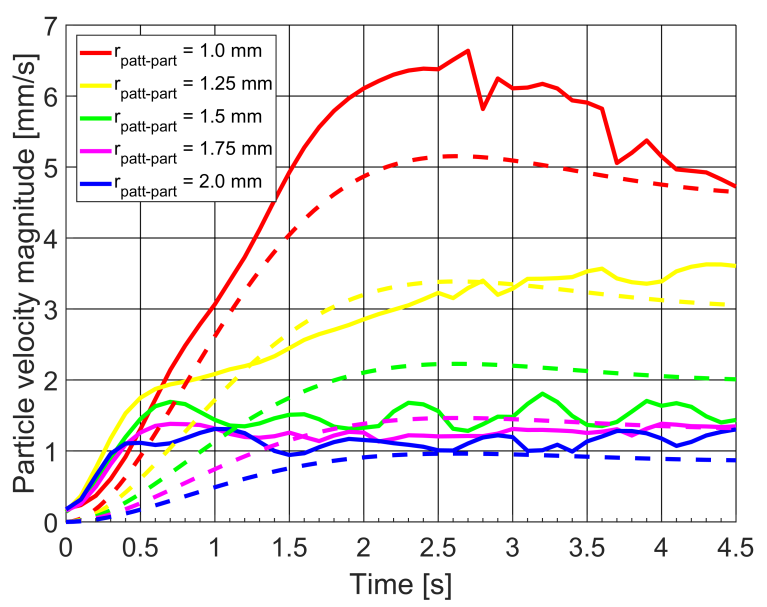

Fig. 7: Particle velocity magnitude $\dot{r}_{\text {part }}$ in function of time. The solid lines correspond to the mean particle velocity measured experimentally and the dashed lines correspond to the estimated velocity using the model given in (1).

The results are shown in Fig. 7 that plots the mean particle velocity in function of time for each $r_{\text {patt-part }}$ value. From those results, a model for the particle velocity in function of the 
pattern-particle is identified in the form: $\dot{r}_{\text {part }}=\hat{\dot{r}}_{\text {part QSS }} \cdot \frac{\dot{\hat{p}}_{\text {part }}}{\hat{r}_{\text {part QS }}}$. The estimated particle velocity at quasi steady-state $\hat{\dot{r}}_{\text {part }}$ QSS model is given by:

$$
\hat{\dot{r}}_{\text {part QSS }}=29.93 \mathrm{~mm} / \mathrm{s} \exp \left(-1.678 \mathrm{~s}^{-1} r_{\text {patt-part }}\right)
$$

The dynamics of the particle velocity is modeled as a second order transfer function defined as:

$$
\frac{\dot{r}_{\text {part }}}{\hat{\dot{r}}_{\text {part QSS }}}=\frac{2.1609}{s^{2}+1.7052 s+2.1609}
$$

This model is compared to the experimental results in Fig. 7. Although the model is not perfect, its accuracy is fair enough to use it for closed-loop control.

\section{Circular pattern discussion and definition}

To stabilize the particle in a tolerance region, a circular pattern is proposed. By drawing a circle around the particle, a flow going inwards the laser circular pattern is generated inside the circle which traps the particle. However, this pattern is not very efficient to displace the particle because the generated flow inside the particle is very slow. The reason for this is that the thermal gradient inside the circle is very small. This actuation strategy is be tested in the next section to maintain the particle inside the tolerance region.

The circular pattern is defined as a circle with a radius of $3.22 \mathrm{~mm}$. For this pattern, the pattern position $\mathbf{X}_{\text {patt }}$ is defined at the circle center.

\section{CONTROLled MANIPULATION USING LASER PATTERNS}

\section{A. Control strategy}

Having analyzed these two patterns, the proposed control strategy is shown in Fig. 8. The arc pattern is used during the displacing phase to move the particle towards the target location. The controller used in this phase operates similarly to the one used for laser spots (subsection III-A). The particle velocity magnitude controller is defined as a PI controller with integrator anti-windup. The proportional gain is $K_{\mathrm{p}}=$ 0.456 , the integral gain is $K_{\mathrm{i}}=0.112$ and the anti-windup gain is $K_{\mathrm{t}}=0.37$. The computed $\hat{\dot{p}}_{\text {part QSS }}$ is then transformed into a pattern-particle distance $r_{\text {patt-part }}$ using the inverse of relation (1). Concerning the control of the particle velocity direction, $\theta_{\text {part }}$ tends fast to the laser-particle direction $\theta_{\text {las-part }}$. It is assumed that this relation is equal to the identity and thus an open-loop controller is used only. In mathematical terms, this is the same as setting that the input of the second SISO system $\left(\theta_{\text {corr }}\right)$ to zero which is equivalent to having $\theta_{\text {patt-part }}$ $=\theta_{\text {part }}=\theta_{\text {part-targ }}$.

Once the particle reaches the tolerance region defined as a circle with radius $3.22 \mathrm{~mm}$, the stabilization phase begins. During this phase, the laser pattern is changed to a circular one which is drawn around the target position $\mathbf{X}_{\text {targ }}$. This circular pattern is constantly generated which ensures that the particle remains at the target location.

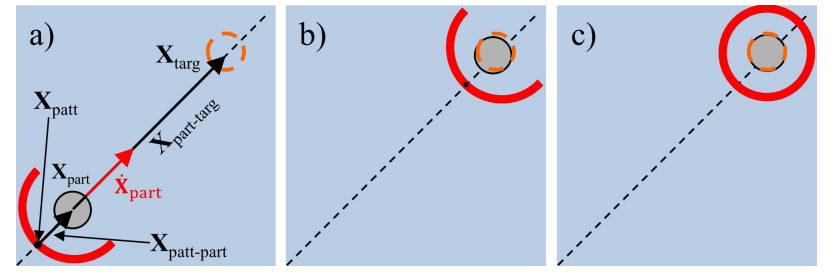

Fig. 8: Control strategy using laser patterns. a-b) Displacing phase: A particle placed at position $\mathbf{X}_{\text {part }}$ is displaced towards a target position $\mathbf{X}_{\text {targ }}$ using an arc laser pattern. This laser pattern generates a flow that goes in the direction of the pattern-particle vector $\mathbf{X}_{\text {patt-part }}$ so it is set to be equal to the direction of vector $\mathbf{X}_{\text {part-targ }}$. This is done until the particle reaches a tolerance region around $\mathbf{X}_{\text {targ }}$. c) Stabilization phase: The laser pattern is changed to a circular one which traps the particle inside the pattern and keeps it still.

\section{B. Preliminary experimental results}

Experiments are performed where a particle is displaced towards a target location. Some pictures of an experiment are shown in Fig. 9 and the analytical results are given in Fig. 10, and should be compared to the ones obtained using point based actuation given in Fig. 3. The error in distance w.r.t. time is shown in Fig. 10a. During the displacing phase, the initial error is around $47 \mathrm{~mm}$. This error reduces as the particle is displaced towards the target location using a laser arc pattern. In terms of the particle velocity (Fig. 10b), it initially accelerates reaching a maximum velocity of $7 \mathrm{~mm} / \mathrm{s}$. As the particle approaches the target location, it begins to decelerate until it reaches the tolerance region.

The laser pattern is then changed into a full circle. As shown in Fig. 10a, this change makes now possible stabilizing the particle inside the tolerance region. In terms of velocity, the particle decelerates very fast (Fig. 10b) and remains almost still with a velocity below $0.2 \mathrm{~mm} / \mathrm{s}$ which is lower than the noise level of $0.8 \mathrm{~mm} / \mathrm{s}$ seen when using the laser spot. In order to show this situation to be stable, the particle was kept more than 5 seconds inside the tolerance region. This was not possible using the laser point where the particle was constantly exiting the tolerance region. It should be noted that a better positioning precision could be attained at the expense of closed-loop control of the position of the center of the circular pattern to precisely position the particle at its target position.

\section{Discussion}

A comparison of the three types of laser patterns (spot, arc, circle) is shown in Table II. The laser spot pattern offers the best performances in terms of maximum velocity and power efficiency. In addition, as it uses less power, it heats the water less which means a smaller temperature increase. The major disadvantages of this pattern are that a controller is required to control the movement direction and that the position stabilization is not possible. So in general terms, it can be said that the laser spot offers the best performance but it is more difficult to implement as it requires a controller 


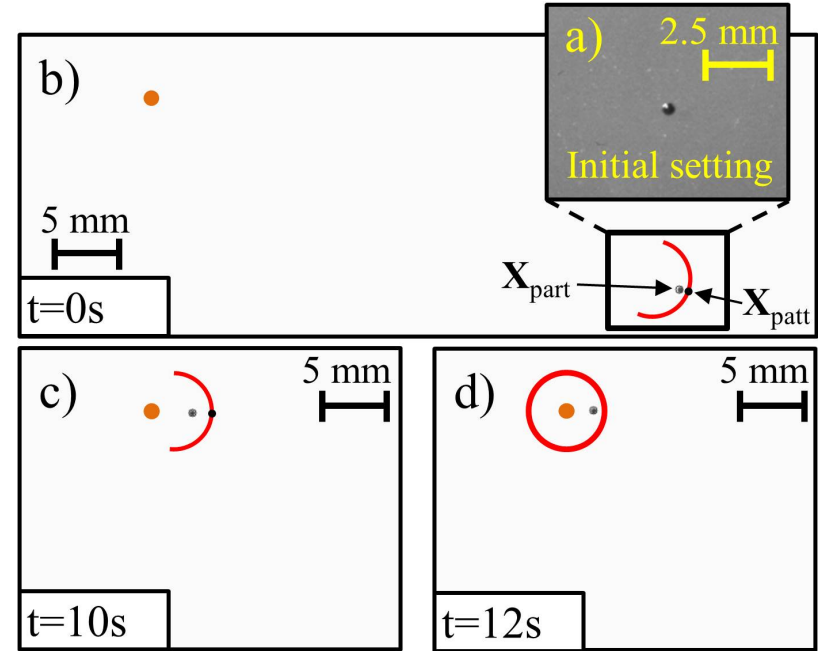

Fig. 9: Controlled manipulation example using laser patterns. a) Zoom of the initial setting: a $500 \mu \mathrm{m}$ diameter AISI 304 steel particle is floating on the water surface with both the particle velocity magnitude and direction controllers turned off. $b$ ) The backlight is turned on and at $t=0 \mathrm{~s}$, both controller are turned on. In the displacement phase, the arc pattern is used to displace the particle from its current position $\mathbf{X}_{\text {part }}$ to the target position $\mathbf{X}_{\text {part }}$ (orange point) by changing the pattern position $\mathbf{X}_{\text {pattern }}(\mathrm{red}$ arc). c) The particle approaches the tolerance region. d) Once the particle is inside it, the pattern is changed to the circular pattern to keep the particle still. The patterns drawn in top of the original pictures.

for the movement direction. The arc pattern allows to impose particle velocities similar to the ones using the laser spot, but it requires up to 3 times larger laser power. As a consequence, the water is heated more which is a drawback as it may damage sensitive components. Its major advantage is that the movement direction control does not require any closed-loop controller like the laser spot, and it is possible to control the direction using an open-loop controller. Same as with the laser spot, the position stabilization is not possible using this technique only. So in general terms, it can be said that the arc patterns offers good performances at the cost of a larger laser power and is easier to implement as the movement direction can be controlled using an open-loop controller. Finally, the circular pattern cannot be used to displace the particle. From the experiments performed, it was seen that it was very difficult to displace the particle. The reason for this is that the flows generated inside the laser pattern are very slow (around the $0.2 \mathrm{~mm} / \mathrm{s}$ ). However, this is the reason why it can be used to stabilize the particle position as once the particle is trapped using this pattern, it will not move anymore. So in general terms, it can be said that the circular pattern is the solution to stabilize the particle position but not to displace it. As conclusion, it can be said that the best strategy to control the particle position would be to use the laser spot or the arc pattern to displace the particle and the circular pattern to stabilize the particle position.

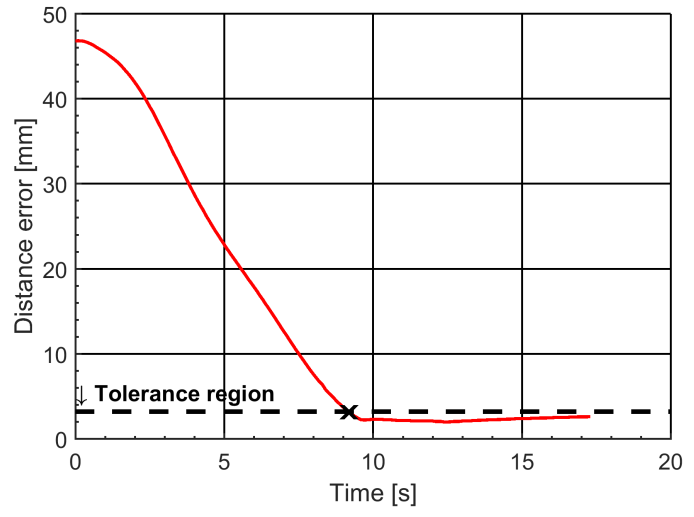

(a) Particle velocity magnitude controller error $r_{\text {error }}$ in function of time.

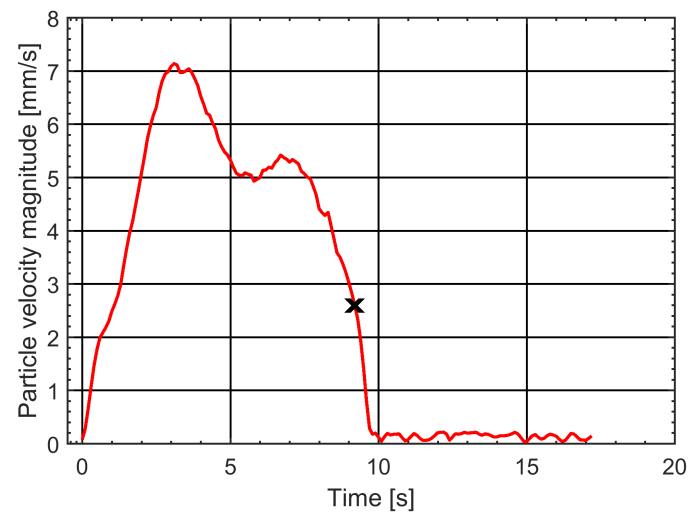

(b) Particle velocity magnitude $\dot{r}_{\text {part }}$ in function of time.

Fig. 10: Results of the closed-loop control of the velocity magnitude of the particle using the control strategy based on laser patterns. Markers are used to show the beginning of the stabilization phase.

TABLE II: Comparison of actuation performances

\begin{tabular}{|l|c|c|c|}
\hline Characteristics & $\begin{array}{c}\text { Circular } \\
\text { pattern }\end{array}$ & $\begin{array}{c}\text { Arc } \\
\text { pattern }\end{array}$ & Laser spot \\
\hline Laser power & $\begin{array}{c}\text { Large (120 } \\
\mathrm{mW})\end{array}$ & $\begin{array}{c}\text { Large }(120 \\
\mathrm{mW})\end{array}$ & $\begin{array}{c}\text { Small }(37 \\
\mathrm{mW})\end{array}$ \\
\hline $\begin{array}{l}\text { Temperature } \\
\text { increase }\end{array}$ & Large & Large & $\begin{array}{c}\text { Small (below } \\
\left.2^{\circ} \mathrm{C}\right)\end{array}$ \\
\hline $\begin{array}{l}\text { Max. particle } \\
\text { Vel. } \\
\text { During control }\end{array}$ & - & $7 \mathrm{~mm} / \mathrm{s}$ & $8.5 \mathrm{~mm} / \mathrm{s}$ \\
\hline $\begin{array}{l}\text { Movement } \\
\text { direction } \\
\text { control }\end{array}$ & - & Simple & $\begin{array}{c}\text { Requires } \\
\text { controller }\end{array}$ \\
\hline $\begin{array}{l}\text { Position } \\
\text { stabilization? }\end{array}$ & Yes & No & No \\
\hline
\end{tabular}




\section{CONCLUSIONS AND FUTURE WORKS}

The use of thermocapillary convective flows generated by laser points and laser patterns is compared for the non-contact micromanipulation of particles. Micrometric size particles placed at the interface between liquid and air are moved toward a target location, and stabilized at their final position. These preliminary results are very promising as they show that the problem of the control of the particle velocity direction as well as the particle position stabilization can be solved using laser patterns.

However this work is only the first step, since additional configurations should be tested to determine the optimal patterns, in term of precision and velocity attained. For example it would be very interesting to estimate the influence of parameters on the dynamics of the system.

\section{ACKNOWLEDGEMENTS}

This research has been funded by the Interuniversity Attraction Poles Programme (IAP 7/38 MicroMAST) initiated by the Belgian Science Policy Office, by the Labex ACTION project (contract "ANR-11-LABX-01-01") and by the Région Franche-Comté. This work has also been carried out in relation with the COST Action MP1106 on Smart and Green Interfaces. The authors thank Prof. Pascal Kockaert and Prof. Pierre Colinet for their valuable scientific advice.

\section{REFERENCES}

[1] M. Sitti, H. Ceylan, W. Hu, J. Giltinan, M. Turan, S. Yim, and E. Diller, "Biomedical applications of untethered mobile milli/microrobots," Proceedings of the IEEE, vol. 103, no. 2, pp. 205-224, 2015.

[2] V. Gauthier, A. Bolopion, and M. Gauthier, "Analytical formulation of the electric field induced by electrode arrays: towards automated dielectrophoretic cell sorting," Micromachines, vol. 8, no. 8, p. 253, 2017.

[3] E. Diller, N. Zhang, and M. Sitti, "Bonding methods for modular micro-robotic assemblies," in IEEE International Conference on Robotics and Automation, 2013, pp. 2588-2593.

[4] A Ashkin, "Forces of a single-beam gradient laser trap on a dielectric sphere in the ray optics regime," Biophysical Journal, vol. 61, no. 2, pp. 569-582, 1992.

[5] M. Yin, E. Gerena, C. Pacoret, S. Haliyo, and S. Régnier, "Highbandwidth $3 \mathrm{~d}$ force feedback optical tweezers for interactive biomanipulation," in 2017 IEEE/RSJ International Conference on Intelligent Robots and Systems (IROS), 2017, pp. 1889-1894.

[6] K. Rajasekaran, E. U. Samani, J. Stewart, and A. G. Banerjee, "Imaging-guided collision-free transport of multiple optically trapped beads," in 2017 International Conference on Manipulation, Automation and Robotics at Small Scales (MARSS), 2017, pp. 1-6.

[7] S. Chowdhury, A. Thakur, P. Švec, C. Wang, W. Losert, and S. K. Gupta, "Automated manipulation of biological cells using gripper formations controlled by optical tweezers," IEEE Transactions on Automation Science and Engineering, vol. 11, no. 2, pp. 338-347, 2014.

[8] F. Arai, K. Yoshikawa, T. Sakami, and T. Fukuda, "Synchronized laser micromanipulation of multiple targets along each trajectory by single laser," Applied Physics Letters, vol. 85, no. 19, pp. 4301-4303, 2004.

[9] K. Onda and F. Arai, "Parallel teleoperation of holographic optical tweezers using multi-touch user interface," in IEEE International Conference on Robotics and Automation, May 2012, pp. 1069 -1074.

[10] H. Chen, C. Wang, X. Li, and D. Sun, "Transportation of multiple biological cells through saturation-controlled optical tweezers in crowded microenvironments," IEEE/ASME Transactions on Mechatronics, vol. 21, no. 2, pp. 888-899, 2016.

[11] S. Hu, S. Chen, S. Chen, G. Xu, and D. Sun, "Automated transportation of multiple cell types using a robot-aided cell manipulation system with holographic optical tweezers," IEEE/ASME Transactions on Mechatronics, vol. 22, no. 2, pp. 804-814, 2017.
[12] H. Yang, X. Li, Y. Wang, G. Feng, and D. Sun, "Control of single-cell migration using a robot-aided stimulus-induced manipulation system," IEEE/ASME Transactions on Mechatronics, vol. 22, no. 2, pp. 815825, 2017.

[13] L. Paterson, M. P. MacDonald, J. Arlt, W. Sibbett, P. E. Bryant, and K. Dholakia, "Controlled rotation of optically trapped microscopic particles," Science, vol. 292, no. 5518, pp. 912-914, 2001.

[14] M. Woerdemann, S. Gläsener, F. Hörner, A. Devaux, L. De Cola, and C. Denz, "Dynamic and reversible organization of zeolite 1 crystals induced by holographic optical tweezers," Advanced Materials, vol. 22, no. 37, pp. 4176-4179, 2010.

[15] S. N. Khonina, V. V. Kotlyar, R. V. Skidanov, V. A. Soifer, K. Jefimovs, J. Simonen, and J. Turunen, "Rotation of microparticles with bessel beams generated by diffractive elements," Journal of Modern Optics, vol. 51, no. 14, pp. 2167-2184, 2004.

[16] M. A. Rykov and R. V. Skidanov, "Modifying the laser beam intensity distribution for obtaining improved strength characteristics of anoptical trap," Appl. Opt., vol. 53, no. 2, pp. 156-164, 2014.

[17] E. Vela, M. Hafez, and S. Regnier, "Laser-induced thermocapillary convection for mesoscale manipulation," International Journal of Optomechatronics, vol. 3, no. 4, pp. 289-302, 2009.

[18] E. Vela, M. Hafez, and S. Régnier, "Contactless automated manipulation of mesoscale objects using opto-fluidic actuation and visual servoing," Review of Scientific Instruments, vol. 85, no. 5, p. 055 107, 2014.

[19] R. Terrazas Mallea, A. Bolopion, J.-C. Beugnot, M. Gauthier, and P. Lambert, "Laser-induced thermocapillary convective flows: a new approach for non-contact actuation at microscale at the fluid/gas interface," IEEE/ASME Transactions on Mechatronics, vol. 22, no. 2, pp. 693-704, 2017.

[20] A. S. Basu and Y. B. Gianchandani, "Virtual microfluidic traps, filters, channels and pumps using marangoni flows," Journal of Micromechanics and Microengineering, vol. 18, no. 11, p. 115031, 2008.

[21] D. Liao, H. Yu, Y. Zhang, and B. Li, "Photothermal delivery of microscopic objects via convection flows induced by laser beam from fiber tip," Appl. Opt., vol. 50, no. 20, pp. 3711-3716, 2011.

[22] W. Hu, K. S. Ishii, and A. T. Ohta, "Micro-assembly using optically controlled bubble microrobots," Applied Physics Letters, vol. 99, no. 9, 094103, 2011.

[23] K. Ishii, W. Hu, and A. Ohta, "Cooperative micromanipulation using optically controlled bubble microrobots," in 2012 IEEE International Conference on Robotics and Automation (ICRA), 2012, pp. 3443-3448.

[24] W. Hu, Q. Fan, and A. T. Ohta, "An opto-thermocapillary cell micromanipulator," Lab Chip, vol. 13, no. 12, pp. 2285-2291, 2013.

[25] _ - "Interactive actuation of multiple opto-thermocapillary flowaddressed bubble microrobots," Robotics and Biomimetics, vol. 1, no. 1, pp. 1-6, 2014.

[26] M. A. Rahman, J. Cheng, Q. Fan, and A. T. Ohta, "Automated actuation of multiple bubble microrobots using computer-generated holograms," in Proceedings of SPIE, vol. 9494, 2015, pp. 1-7.

[27] M. A. Rahman, A. T. Ohta, N. Takahashi, and Z. Wang, "Closed-loop vision-assisted actuation of a bubble microrobot," in 2016 IEEE 10th International Conference on Nano/Molecular Medicine and Engineering (NANOMED), 2016, pp. 61-64.

[28] M. A. Rahman, J. Cheng, Z. Wang, and A. T. Ohta, "Cooperative micromanipulation using the independent actuation of fifty microrobots in parallel," Scientific Reports, vol. 7, p. 3278, 2017.

[29] R. Terrazas Mallea, A. Bolopion, J.-C. Beugnot, M. Gauthier, and P. Lambert, "Closed-loop particle motion control using laser-induced thermocapillary convective flows at the fluid/gas interface at micrometric scale," IEEE/ASME Transactions on Mechatronics, In press, 2018.

[30] — - "1d manipulation of a micrometer size particle actuated via thermocapillary convective flows," in 2017 IEEE/RSJ International Conference on Intelligent Robots and Systems (IROS), 2017, pp. 408413.

[31] A. Rapacchietta and A. Neumann, "Force and free-energy analyses of small particles at fluid interfaces:ii. spheres," Journal of Colloid and Interface Science, vol. 59, no. 3, pp. 555 -567, 1977. 